\title{
DIMENSIONAMENTO DE UM SISTEMA DE EXAUSTÃO PARA UMA CÉLULA DE SOLDAGEM DE UMA INDÚSTRIA DE MÁQUINAS AGRÍCOLAS
}

\author{
Bruna Karine dos Santos ${ }^{1 *}$, Diego Michael Cornelius dos Santos ${ }^{2}$, César Gabriel dos Santos ${ }^{3}$ \\ 1 Programa de Pós-Graduação em Engenharia Agrícola, UFSM, 97105-900, Santa Maria, Brasil. \\ 2 Programa de Pós-Graduação em Engenharia Mecânica, UFSM, 97105-900, Santa Maria, Brasil. \\ $3^{3}$ Departamento de Engenharia Mecânica, UFSM - Campus de Cachoeira do Sul, 96506-322, Cachoeira do Sul, Brasil.
}

*E-mail: bruninhaa_hz@hotmail.com

Recebido em:16/02/2019 Aceito em:30/07/2019

\section{RESUMO}

A união de materiais por meio do processo de soldagem GMAW é amplamente utilizada na indústria de máquinas agrícolas devido às suas características, que permitem bom nível de produtividade e de desempenho do processo. Porém, sua utilização resulta na geração de poluentes nocivos à saúde humana, também conhecidos como fumos metálicos ou gases tóxicos. A inalação dessas partículas pode causar problemas de saúde, como a diminuição das funções pulmonares, além de contribuir para o surgimento de diversas doenças. Desta forma, é necessário utilizar um sistema de captação e transporte dessas partículas para evitar o contato direto entre os gases tóxicos e os operadores envolvidos no processo. Neste contexto, este trabalho tem como objetivo determinar uma metodologia que permita realizar o dimensionamento de um sistema de captação e movimentação dessas partículas, desde a escolha do captor até a definição do tipo de ventilador a ser utilizado, assim como suas respectivas características. De acordo com o memorial de cálculo abordado neste trabalho, foi possível determinar o ventilador ideal para esta situação, levando em consideração as singularidades utilizadas nos dutos, o tipo de contaminante e a perda de carga resultante da coleta e transporte dos gases tóxicos originados pelo processo de soldagem.

Palavras-chave: Fumos metálicos. Sistema de ventilação. Ventilação forçada. Processo de soldagem.

\section{Introdução}

Através do processo de soldagem é possível obter a união de componentes e conjuntos metálicos, utilizando uma fonte de calor que é responsável por fundir ambos os materiais [1-2]. O processo de soldagem Gas Metal Arc Welding (GMAW) se caracteriza por utilizar um eletrodo e gás de proteção, e consiste em um processo de soldagem amplamente utilizado para unir componentes na indústria agrícola [3].

Além de permitir a união de componentes, o processo de soldagem apresenta alguns resultados indesejáveis, como a geração de poluentes nocivos à saúde humana, conhecidos como fumos metálicos e gases tóxicos [4-5-6]. O contato dos soldadores com a fumaça resultante do processo de soldagem contribui para a diminuição das funções pulmonares e o surgimento de algumas doenças, como, por exemplo, pneumoconiose, intoxicação de manganês, febre de fumaça de solda, entre outros efeitos envolvendo o sistema nervoso central e os olhos [5-7]. Esses poluentes são classificados como agentes de alta prioridade, pois sua composição é formada por óxidos metálicos (ferro, alumínio, cromo, níquel, manganês), compostos inorgânicos (fluoretos e silicatos não cristalinos) e gases (ozônio, óxidos de nitrogênio e monóxido de carbono) [5-7].

A emissão dos gases tóxicos e de fumos de soldagem está diretamente ligada a alguns parâmetros de soldagem como frequência de transferência, corrente elétrica, tempo de arco aberto, gás de proteção, entre outros fatores relacionados ao material e a seu tratamento superficial [6].

Quando as indústrias utilizam somente ventilação natural com poucas correntes de ar nas áreas de soldagem, os gases e fumos originados neste processo ficam concentrados no ambiente industrial e contribuem para a contaminação dos colaboradores. Uma alternativa para reduzir esse problema é a aplicação de equipamentos de ventilação forçada, que evitam a dispersão dos contaminantes por meio da sua captação e transporte, minimizando os riscos e garantindo melhores condições de trabalho aos soldadores [5]. A aplicação desse tipo de sistema é indicada para ambientes fechados ou que apresentam poucas correntes de ar, onde a ventilação mecânica é realizada por meio de ventiladores, evitando a dispersão dos contaminantes e 


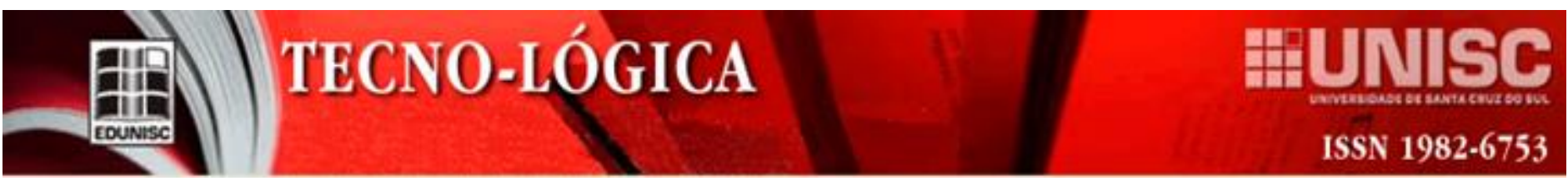

permitindo a sua retirada do ambiente de trabalho, diminuindo consideravelmente os danos à saúde do ser humano [8-9].

Com base nessas informações, este trabalho tem como objetivo determinar uma metodologia que permita realizar o dimensionamento de um sistema de captação e movimentação de partículas, desde a escolha do captor até a definição do tipo de ventilador, assim como tubos, conexões e reduções.

\subsection{Processo de Soldagem GMAW}

A Soldagem GMAW é denominada como o processo de união entre duas partes metálicas, usando uma fonte de calor que é responsável por fundir ambos os materiais [2]. Caracteriza-se por ser empregada na união de componentes de estruturas metálicas e equipamentos nos mais variados campos de aplicação, incluindo a construção naval, estruturas civis, vasos de pressão, tubulações, usinas hidrelétricas e diversos equipamentos [10].

A soldagem a arco elétrico é definida como uma descarga elétrica que é mantida através de um gás ionizado, iniciada por uma quantidade de elétrons emitidos do eletrodo negativo aquecido e mantido pela ionização térmica do gás. Com isso, ocorre o surgimento da poça de fusão, resultando na união entre as duas partes metálicas [2].

Outra peculiaridade deste processo está relacionada à utilização de um gás de proteção, que pode ser ativo ou inativo, e de um eletrodo (no caso do eletrodo revestido, não se utiliza gás de proteção). $\mathrm{O}$ eletrodo consiste em um arame que é consumido por meio do arco elétrico, resultando na fusão de ambos os materiais. Esse processo de soldagem é muito utilizado para unir materiais na indústria de máquinas e implementos agrícolas [3].

Como resultado desse processo, ocorre a produção de elementos nocivos à saúde humana, tais como fumos metálicos, gases, partículas e radiação. A geração de fumos metálicos no processo de soldagem GMAW caracteriza-se por estar diretamente ligada às características da transferência metálica (frequência de transferência, tempo de arco aberto e volume da poça de fusão), corrente elétrica e gás de proteção [11].

\subsection{Contaminantes}

O profissional responsável por realizar a soldagem dos componentes está constantemente exposto aos mais variados riscos durante a realização de suas atividades, dentre os quais se destacam os agentes físicos, químicos, biológicos e ergonômicos [12]. Além disso, o processo de soldagem utiliza altas temperaturas e radiação ultravioleta, resultando na geração elevada de gases venenosos próximos da área do arco elétrico.

Neste contexto, em relação aos riscos químicos, os elementos encontrados nos fumos de soldagem são classificados como agentes de alta prioridade, os quais possuem óxidos metálicos de ferro, alumínio, cromo, níquel, manganês, compostos inorgânicos como fluoretos e silicatos não cristalinos, além de gases como o ozônio, óxidos de nitrogênio e monóxido de carbono [7].

A formação dos fumos metálicos causa danos à saúde, dentre os quais se destacam a pneumoconiose, a intoxicação de manganês, a febre de fumaça de solda, os efeitos sobre o sistema nervoso central e os olhos, entre outros efeitos indesejáveis [137]. Portanto, recomenda-se que a operação de soldagem seja efetuada em locais bem ventilados e, se necessário, com a utilização de ventiladores e exaustores para realizar a captação e a respectiva dispersão dos resíduos para fora do ambiente industrial [14].

\subsection{Sistema de ventilação}

O sistema de ventilação é muito utilizado em ambientes fechados ou que apresentam poucas correntes de ar em seu interior, sendo necessária a retirada ou fornecimento de ar a determinado ambiente. Além disso, este tipo de sistema pode evitar a dispersão de contaminantes no ambiente industrial, garantindo que homem e máquina convivam em um mesmo recinto sem causar danos à saúde do ser humano [9].

Dentre os diversos tipos de ventilação (exaustora, natural ou diluidora) utilizada na indústria, a ventilação local exaustora é realizada de forma mecânica e se destaca por extrair o contaminante no próprio local em que ele é produzido, antes mesmo de se espalhar pelo ambiente. Esse tipo de ventilação atua capturando os poluentes por meio de uma corrente de ar com velocidade adequada (velocidade de captura), criada por meios de dispositivos especiais, também conhecidos como captores. A velocidade do ar nas canalizações de ventilação local exaustora deve ser suficiente para manter as partículas do contaminante em suspensão, ao mesmo tempo em que elas devem ser transportadas. Além disso, também é responsável por transportar pelo ar detritos como poeiras, fumos metálicos e vapores [8].

Os principais componentes de um sistema de ventilação exaustora são [15]:

- Captor: é o ponto de entrada dos gases a serem exauridos pelo sistema e está diretamente ligado à velocidade de captação $(\mathrm{Vc})$;

- Sistema de dutos: responsável pelo transporte dos gases captados;

- Ventilador: responsável pela variação de pressão entre o captor e a saída do sistema, movimentando os gases por meio da tubulação.

É importante salientar que os dutos do sistema de ventilação local exaustora devem possuir seção circular, sempre que possível, a fim de evitar arestas ou zonas de velocidade reduzida, que possibilitam a estagnação dos contaminantes [8]. 


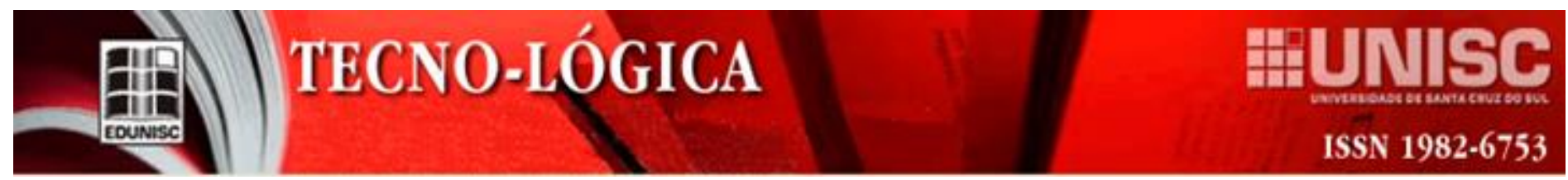

Como o sistema de exaustão possui uma série de tubos e elementos, a perda de carga deve ser considerada, pois ela ocorre por meio do atrito entre o gás e as paredes internas da tubulação, convertendo a energia mecânica em energia térmica. $\mathrm{O}$ resultado desse atrito causa a diminuição no nível de pressão total do sistema, o qual deverá ser compensado posteriormente [16].

Além da perda de carga pelo atrito entre o fluido e o duto, as singularidades utilizadas ao longo da tubulação também devem ser consideradas. Essas singularidades (cotovelos, junções, curvas e reduções) causam distúrbios no fluxo do ar, além de representarem pontos em que ocorrem perda de carga localizada. Esses pontos devem ser projetados de forma que a geometria apresentada ocasione a menor perda de carga possível no sistema $[17-18]$.

\section{Metodologia}

O trabalho foi desenvolvido em cinco etapas, conforme ilustrado na Figura 1. A revisão bibliográfica foi elaborada por meio de pesquisa em livros e artigos publicados em revistas, por meio dos quais foi possível realizar o levantamento de informações sobre os fumos metálicos e a metodologia referente ao memorial descritivo a ser utilizado. Em relação à coleta dos dados, foi realizada uma visita a uma empresa do setor de metal mecânico, onde foi definida a quantidade de equipamentos de soldagem e a distância entre eles, de forma que fossem definidas as dimensões que o sistema deve possuir.

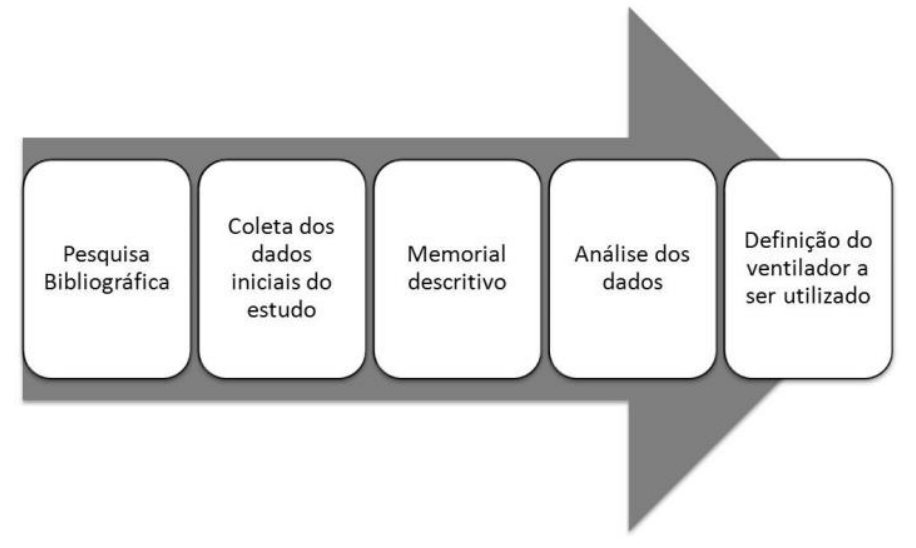

Figura 1 - Etapas do desenvolvimento do trabalho.

O dimensionamento do sistema de ventilação exaustora foi realizado conforme a metodologia abordada por Costa [8]. No memorial descritivo foram definidas as vazões e velocidades do gás em cada ponto de estrangulamento, além de determinar as perdas de cargas na tubulação e nas singularidades. A etapa final, relacionada à escolha do ventilador a ser utilizado, foi realizada em função da análise dos dados calculados em relação ao catálogo do fabricante de ventilador. Essa escolha considerou os seguintes parâmetros: perda de carga, potência necessária e vazão total utilizada pelo sistema.

\subsection{Memorial descritivo}

O memorial descritivo define as etapas realizadas para dimensionar o sistema de exaustão, que foi realizado seguindo o método proposto por Costa [8]. Neste método, inicialmente foi necessário que um especialista realizasse uma avaliação do local onde é realizada a soldagem, permitindo definir o tipo de captor a ser utilizado, assim como a respectiva área do bocal $\left(\Omega_{0}\right)$ responsável pela coleta dos contaminantes. O bocal do captor pode ser definido conforme ilustrações da Figura 2.

\begin{tabular}{|c|c|c|c|}
\hline Tipo de captor & Descrição & $\lambda$ & $\mu$ \\
\hline$\longrightarrow$ & Extremidade plana de duto & 0,93 & 0,72 \\
\hline$\longrightarrow$ & Boca bem arredondada & 0,04 & 0,98 \\
\hline & $\begin{array}{c}\text { Extremidade de duto } \\
\text { flangeado }\end{array}$ & 0,49 & 0,82 \\
\hline & $\begin{array}{c}\text { Captor ligado ao duto por } \\
\text { peça cônica circular }\end{array}$ & 0,15 & 0,93 \\
\hline & $\begin{array}{c}\text { Captor ligado ao duto por } \\
\text { peça arredondada }\end{array}$ & $0,06-0,10$ & 0,97 \\
\hline
\end{tabular}

Figura 2 - Tipos de captores. Adaptada de Costa [8].

A escolha do tipo de captor está relacionada ao coeficiente de entrada do gás $(\mu)$ e ao coeficiente de atrito $(\lambda)$, que variam de acordo com a forma. Ambos influenciam a queda de pressão no sistema.

A distância do ponto de operação até o bocal do captor (x) é outro parâmetro que foi considerado. Essa distância pode ser determinada conforme informações apresentadas na Tabela 1.

Tabela 1 - Distância do ponto de operação até o captor.

\begin{tabular}{ccc}
\hline Distância $(\mathbf{x})[\mathbf{m}]$ & $\begin{array}{c}\text { Duto simples }(\mathbf{Q}) \\
{\left[\mathbf{m}^{\mathbf{3}} \cdot \mathbf{s}^{\mathbf{- 1}}\right]}\end{array}$ & $\begin{array}{c}\text { Duto com entrada flangeada } \\
\text { ou cônica }(\mathbf{Q})\left[\mathbf{m}^{\mathbf{3}} \cdot \mathbf{s}^{-\mathbf{1}}\right]\end{array}$ \\
\hline 0,152399 & 0,158100 & 0,117980 \\
0,152399 a 0,228600 & 0,356310 & 0,264290 \\
0,228600 a 0,304800 & 0,630040 & 0,471900 \\
\hline
\end{tabular}

Fonte: Adaptada de Macintyre [17].

O próximo parâmetro definido se trata do coeficiente de forma da boca do captor (k), obtido por meio da Equação 1. 


\section{㭏) TECNO-LÓGICA

$$
k=\frac{0,1 . \Omega_{0}+x^{2}}{0,1 . \Omega_{0}} \text { [Adimensional] }
$$

De acordo com o tipo de partícula foi definida a velocidade de captura (Vc) dos contaminantes em função da operação realizada, como pode ser analisado na Tabela 2 .

Tabela 2 - Velocidade de captura em função da operação.

\begin{tabular}{ccc}
\hline Operação & $\mathbf{V}_{\mathbf{C}}\left(\mathbf{m . s}^{\mathbf{- 1}}\right)$ & Observação \\
\hline Jatos abrasivos & 2,5 & Em cabines \\
\hline Enchimento de barris & 0,4 a 0,5 & No ponto de operação \\
\hline Corte manual de granito & 1,0 & No ponto de operação \\
\hline Esmerilhamento & 1,0 a 2,0 & Grelha de fluxo descendente \\
\hline Pintura a pistola & 0,5 a 1,0 & Face da cabine \\
\hline Solda de prata & 0,5 & Face da coifa \\
\hline Solda elétrica & 0,5 a 1,0 & No ponto de operação
\end{tabular}

Fonte: Adaptada de Costa [8].

Com os parâmetros acima já definidos, de acordo com a necessidade, foi possível determinar a vazão $(\mathrm{Q})$ necessária no bocal escolhido. Este cálculo foi realizado por meio da utilização da Equação 2.

$$
\mathrm{Q}=\mathrm{k} . \mathrm{Vc} \cdot \Omega_{0}\left[\mathrm{~m}^{3} \cdot \mathrm{s}^{-1}\right]
$$

A velocidade de transporte (c) está diretamente ligada ao tipo de partícula presente no sistema. Portanto, a velocidade foi definida de acordo com as informações apresentadas na Tabela 3.

Tabela 3 - Velocidade de ar em dutos.

\begin{tabular}{lc}
\hline \multicolumn{1}{c}{ Tipo de contaminante } & c $\left(\mathbf{m . s}^{\mathbf{1}}\right)$ \\
\hline Vapores, gases, fumos, poeiras muito finas $(<0,05 \mathrm{~mm})$ & 10 \\
\hline Poeiras secas finas & 15 \\
\hline Poeiras industriais médias & 17,5 \\
\hline Partículas grossas & 17,5 a 22,5 \\
\hline Partículas grandes, material úmido. & $>22,5$ \\
\hline
\end{tabular}

Fonte: Adaptada de Costa [8].

Com base na equação da continuidade (Equação 3) foi possível determinar a área da seção transversal (A) dos dutos e, a partir deste valor, determinou-se o diâmetro (D) de cada duto utilizado no sistema, de acordo com a Equação 4.

$$
\begin{gathered}
Q=A . c \\
A=\frac{\pi \cdot D^{2}}{4} \quad[\mathrm{~m}]
\end{gathered}
$$

Sendo que:

$\mathrm{D}=$ diâmetro $(\mathrm{m})$.

$\pi=\operatorname{Pi}[3,1415]$.
Para determinar a perda de carga no sistema, foi necessário definir os valores referentes ao coeficiente de atrito $(\lambda)$ de cada uma das curvas e joelhos utilizados no sistema de ventilação, como pode ser observado na Tabela 4 e na Tabela 5.

Tabela 4 - Coeficiente de atrito nas curvas das canalizações.

\begin{tabular}{ccccccccccc}
\hline $\mathrm{R} / \mathrm{D}$ & 0 & 0,5 & 0,75 & 1,0 & 1,5 & 2,0 & 3,0 & 4,0 & 5,0 \\
\hline & \multicolumn{1}{c}{0,87} & 0,73 & 0,38 & 0,26 & 0,17 & 0,15 & 0,14 & 0,14 & 0,16 \\
\hline
\end{tabular}

Tabela 5 - Coeficiente de atrito dos acessórios.

\begin{tabular}{cc}
\hline Acessórios & $\boldsymbol{\lambda}$ \\
\hline Joelho de seção circular & 0,87 \\
Joelho de seção retangular & 1,25 \\
Com veias simples & 0,35 \\
Com veias duplas & 0,10 \\
\hline
\end{tabular}

Fonte: Adaptada de Costa [8].

Outra perda de carga que ocorre nesse tipo de sistema é atribuída ao ângulo da diminuição do diâmetro em cada uma das seções, sendo que o coeficiente de atrito pode ser definido conforme a Tabela 6 .

Tabela 6 - Coeficiente de atrito de redução de diâmetro.

\begin{tabular}{cc}
\hline Ângulo $(\alpha)$ & $\boldsymbol{\lambda}$ \\
\hline $5^{\circ}$ & 0,28 \\
\hline $10^{\circ}$ & 0,44 \\
\hline $15^{\circ}$ & 0,58 \\
\hline $20^{\circ}$ & 0,72 \\
\hline $25^{\circ}$ & 0,87 \\
\hline $30^{\circ}$ & 1,00 \\
\hline$>30^{\circ}$ & 1,00
\end{tabular}

Fonte: Adaptada de Macintyre [17].

Com a determinação dos parâmetros envolvendo a perda de carga do sistema, são iniciados os cálculos referentes à definição da perda total de carga que o sistema irá apresentar, utilizando-se da Equação 5.

$$
J=\Sigma \lambda \frac{V r^{2}}{2 g} \cdot \gamma
$$

Onde:

$J=$ Perda de carga $\left[\mathrm{kg} \cdot \mathrm{m}^{-2}\right]$.

$V r=$ Velocidade real $\left[\mathrm{m} . \mathrm{s}^{-1}\right]$.

$\Sigma \lambda=$ Somatório do coeficiente de atrito [adimensional].

$g=$ Intensidade do campo gravitacional $\left[9,81 \mathrm{~m} \cdot \mathrm{s}^{-2}\right]$.

$\gamma=$ Peso específico do ar $\left[1,2 \mathrm{~kg} \cdot \mathrm{m}^{-3}\right]$. 


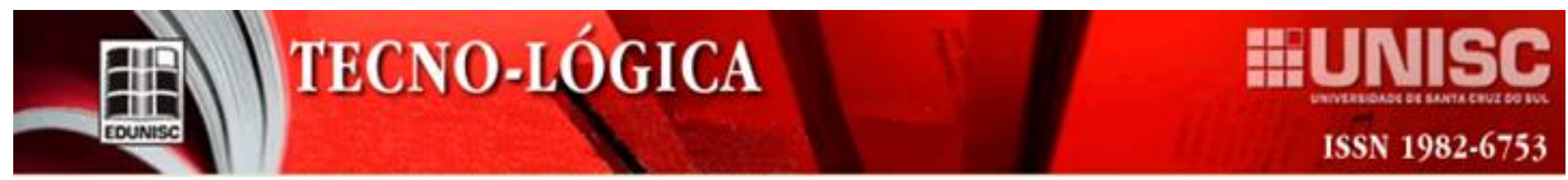

Por meio da Equação 6, foi determinada a perda de carga referente ao contato do fluido com as paredes internas da tubulação.

$$
J_{d u t o s}=0,001026 \cdot l \cdot \frac{V r^{2,51}}{Q^{0,61}}
$$

Onde:

$\mathrm{J}_{\text {dutos }}=$ Perda de carga dos dutos $\left[\mathrm{kg} \cdot \mathrm{m}^{-2}\right]$.

$\mathrm{l}=$ Comprimento da canalização $[\mathrm{m}]$.

Com a definição dos parâmetros e o desenvolvimento dos cálculos realizados é possível determinar o ventilador que melhor se enquadra na necessidade da empresa. Essa escolha é realizada em função do catálogo da Otam [19]. Neste caso, é aconselhado incrementar a potência lida nas curvas do gráfico em $20 \%$, a fim de compensar as perdas de transmissão ou uma possível sobrecarga do sistema [19].

\section{Resultados e discussões}

Após a realização da visita à empresa, foram elaboradas as medições necessárias para realizar o dimensionamento do sistema, como pode ser analisado na Figura 3. Vale salientar que na Figura 3 os dutos verticais não estão visíveis, e serão abordados posteriormente.

Inicialmente, foi estabelecido que a área do captor utilizada para realizar a coleta dos contaminantes será igual em todas as fontes de soldagem, sendo considerado um quadrado com uma área em cada captor que será de $0,35 \mathrm{~m}^{2}\left(\Omega_{0}\right)$.

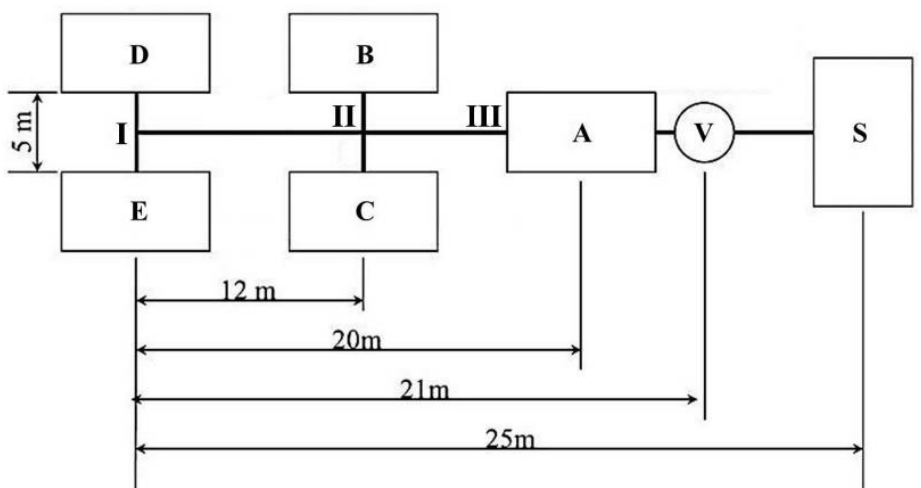

Figura 3 - Localização das máquinas de soldagem. Na imagem é possível identificar as seguintes partes do sistema: Captor (A, B, C, D, E), Ventilador (V), Saída de ar (S) e redução da seção (I, II, III).

Dentre os captores ilustrados na Figura 2, foi escolhido o captor "Boca bem arredondada", por apresentar baixo coeficiente de atrito e alto coeficiente de entrada, se comparado aos demais tipos de captores. Em relação à distância do captor até o ponto de operação da soldagem, considerou-se a distância de 0,3048 m, de acordo com as informações da Tabela 1. Com essas informações definiu-se o coeficiente de forma $(\mathrm{k})$ da boca do captor, por meio da Equação 1:

$$
\mathrm{k}=3,654
$$

Com o valor do coeficiente de forma da boca do captor determinado, determinou-se a vazão de cada bocal, por meio da Equação 2. Os parâmetros envolvidos nesse cálculo foram: a velocidade de captura da soldagem elétrica $\left(0,5 \mathrm{~m} \cdot \mathrm{s}^{-1}\right)$, o coeficiente de forma $(3,654)$ e a área de coleta de cada captor $(0,35$ $\left.\mathrm{m}^{2}\right)$.

$$
Q=0,64 \mathrm{~m}^{3} \cdot \mathrm{s}^{-1}
$$

Após determinar a vazão em cada bocal, definiu-se que a velocidade máxima (c) nos diversos trechos da tubulação foi de 10 $\mathrm{m} . \mathrm{s}^{-1}$, conforme informações apresentadas na Tabela 3. No momento em que a vazão $(\mathrm{Q})$ é definida em cada segmento da tubulação, é possível determinar a área (A) por meio da Equação 3 , isolando-se a variável "A". Na sequência, o diâmetro (D) dos dutos foi calculado com base na Equação 4.

Como dificilmente o diâmetro calculado é encontrado comercialmente, foi necessário realizar pesquisas em catálogos de fabricantes, a fim de encontrar tubos em que o diâmetro comercial (Dc) seja similar ao diâmetro calculado (D). Porém, se o Dc for menor que o $\mathrm{D}$, a velocidade do contaminante será maior do que a calculada ou vice-versa. Portanto, foi necessário calcular a velocidade real $\left(\mathrm{V}_{\mathrm{R}}\right)$ de captura dos contaminantes, por meio da Equação 3, isolando-se a variável "c" passando a ser denominada de " $V_{R}$ ". O resultado dos diâmetros comerciais e de suas respectivas velocidades pode ser observado na Tabela 7 .

Tabela 7 - Definição dos diâmetros e das velocidades.

\begin{tabular}{ccccccc}
\hline Dutos & $\mathbf{Q}\left[\mathbf{m}^{3} \mathbf{s}^{-1}\right]$ & $\mathbf{A}\left[\mathbf{m}^{2}\right]$ & $\mathbf{C}\left[\mathbf{m} . \mathbf{s}^{-1}\right]$ & $\mathbf{D}[\mathbf{m}]$ & $\mathbf{D}_{\mathbf{c}}[\mathbf{m}]$ & $\mathbf{V}_{\mathbf{R}}\left[\mathbf{m} . \mathbf{s}^{-1}\right]$ \\
\hline $\begin{array}{c}\text { I-D, I-E, II- } \\
\text { B, II-C, III-A }\end{array}$ & 0,640 & 0,064 & 10 & 0,285 & 0,260 & 12,020 \\
\hline II-I & 1,280 & 0,128 & 10 & 0,404 & 0,394 & 10,510 \\
\hline II-III & 2,560 & 0,256 & 10 & 0,571 & 0,546 & 10,930 \\
\hline S-V, V-III & 3,200 & 0,320 & 10 & 0,638 & 0,635 & 10,100 \\
\hline
\end{tabular}

Para determinar os diâmetros comerciais dos dutos, apresentados na Tabela 7, utilizou-se o catálogo da empresa Tubos Oliveira [20], o qual se baseia na norma NBR 5590 (ASTM A106/ API - 5L).

A próxima etapa consistiu na determinação da perda de carga presente no sistema, em relação ao captor, curvas, joelhos, redução de seção e saída do fluído. Para isso, foram desenvolvidos cálculos utilizando a Equação 5, de modo que os resultados fossem apresentados conforme a Tabela 8. 


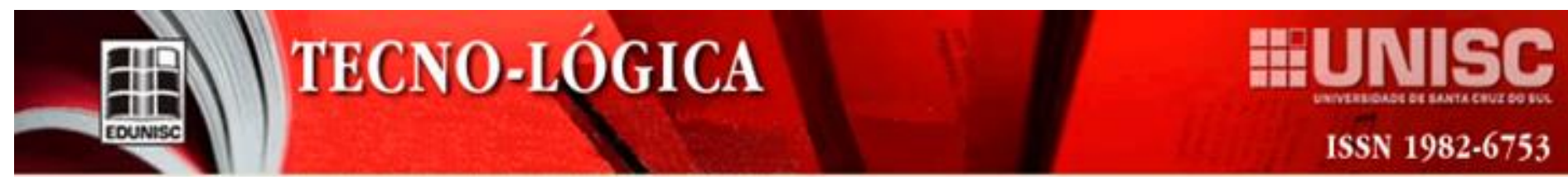

Tabela 8 - Perda de carga nos acessórios.

\begin{tabular}{ccc}
\hline Elemento & $\boldsymbol{J}\left(\mathbf{k g . m ^ { - 2 }}\right)$ & $\boldsymbol{J}(\mathbf{P a})$ \\
\hline Curvas & 4,51 & 44,21 \\
\hline Captor & 0,87 & 8,50 \\
\hline Joelho & 18,86 & 184,92 \\
\hline Saída & 5,77 & 56,59 \\
\hline Redução da seção & 18,78 & 184,21 \\
\hline Total & 48,79 & 478,44 \\
\hline
\end{tabular}

O sistema possui quatro curvas, onde utilizou-se 0,26 como coeficiente de atrito, conforme ilustrado na Tabela 4. Foram utilizados cinco captores e, de acordo com a Figura 2, os captores possuem coeficiente de atrito de 0,04 .

Em relação aos cinco joelhos utilizados no sistema, tomouse o coeficiente de atrito de 0,87 , informado na Tabela 5 . Além disso, foram consideradas sete reduções de seção, que apresentam ângulo de $15^{\circ}$ e que, de acordo com a Tabela 6 , possuem um coeficiente de atrito de 0,58 . Por fim, para a saída do fluido, utilizou-se um coeficiente de atrito de 1 , conforme sugestão de Costa [8].

Outro fator a ser levado em consideração é a perda de carga que ocorre entre o fluido e a superfície interna da tubulação, a qual poderá ser calculada de acordo com a Equação 6. As informações abordadas na Tabela 9 permitem o cálculo da perda de carga decorrente do comprimento da tubulação utilizada no sistema.

Tabela 9 - Perda de carga na tubulação.

\begin{tabular}{cccccc}
\hline Segmento & $\mathbf{Q}\left[\mathbf{m}^{\mathbf{3}} \cdot \mathbf{s}^{-\mathbf{1}}\right]$ & $\mathbf{L}[\mathbf{m}]$ & $\mathbf{V}_{\mathbf{R}}\left[\mathbf{m} \cdot \mathbf{s}^{-\mathbf{1}}\right]$ & $\boldsymbol{J}\left(\mathbf{k g} \cdot \mathbf{m}^{-\mathbf{2}}\right)$ & $\boldsymbol{J}(\mathbf{P a})$ \\
\hline V-III & 3,20 & 1 & 9,712 & 0,15 & 1,49 \\
\hline S-V & 3,20 & 4 & 9,712 & 0,61 & 5,95 \\
\hline III-II & 2,56 & 8 & 9,136 & 1,19 & 11,70 \\
\hline II-I & 1,28 & 12 & 8,256 & 2,12 & 20,78 \\
\hline I-D & 0,64 & 10 & 8,417 & 2,83 & 27,74 \\
\hline I-E & 0,64 & 10 & 8,417 & 2,83 & 27,74 \\
\hline II-B & 0,64 & 10 & 8,417 & 2,83 & 27,74 \\
\hline II-C & 0,64 & 10 & 8,417 & 2,83 & 27,74 \\
\hline III-A & 0,64 & 7,5 & 8,417 & 2,12 & 20,81 \\
\hline & & Total & & 17,51 & 171,68 \\
\hline
\end{tabular}

Com o desenvolvimento da Tabela 8 e da Tabela 9, foi possível determinar a perda de carga total do sistema, que totaliza $66,3 \mathrm{~kg} \cdot \mathrm{m}^{-2}$ e corresponde a 650,12 Pa.

Após a determinação da vazão total necessária, bem como do tipo de captor, dutos e perdas de carga no sistema, foi recomendado através do catálogo o ventilador adequado para o estudo de caso. Para isso, foram utilizados os valores referentes à perda de carga e à vazão total utilizada pelo sistema. A perda de carga total $(650,12 \mathrm{~Pa})$ teve que ser convertida em milímetro coluna de água (mmca).

Desta forma, analisando o catálogo, concluiu-se que o ventilador mais adequado é o modelo RFS 710 classe I, por apresentar melhor rendimento, em torno de $88 \%$, se comparado com os demais. A escolha pode ser visualizada na Figura 4, onde os parâmetros utilizados estão identificados.

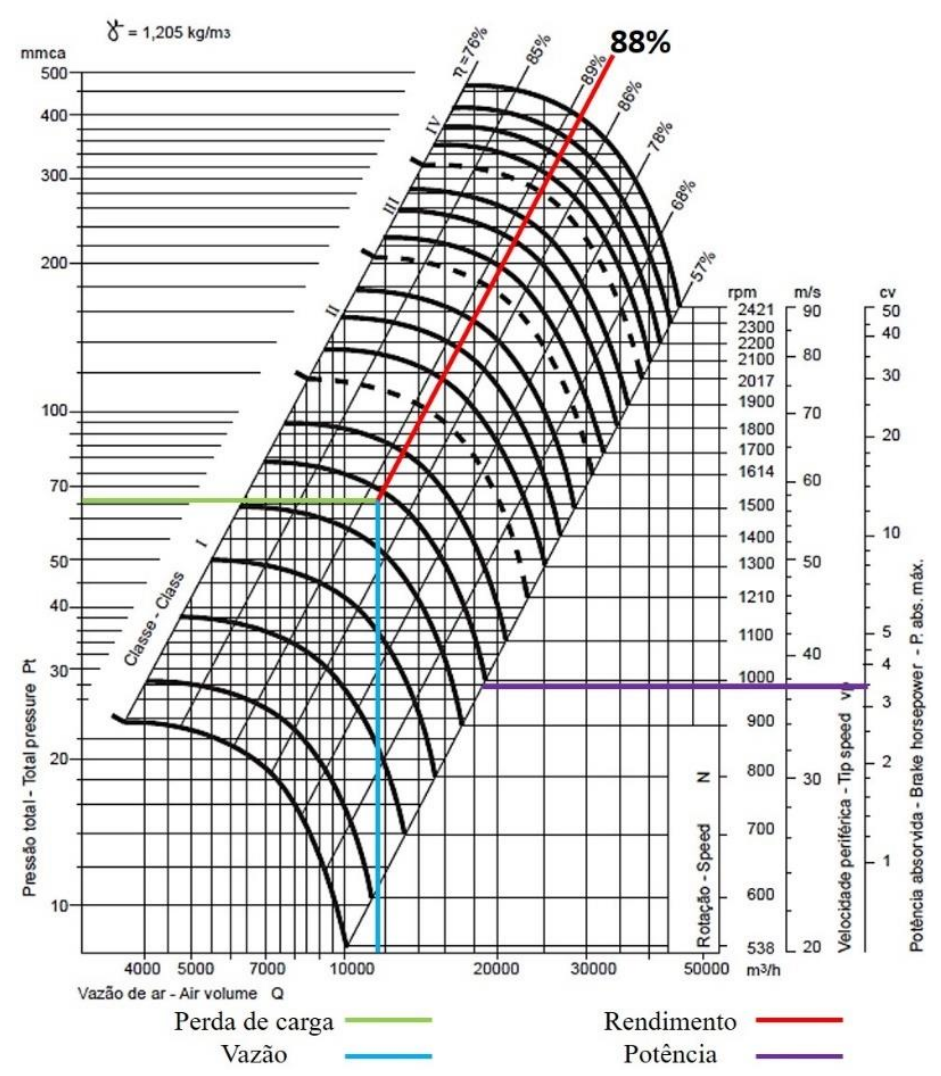

Figura 4 - Ventilador escolhido. Adaptada de OTAM [19].

Seguindo a recomendação do catálogo [19], a potência de $3,5 \mathrm{cv}$, encontrada no gráfico da curva do ventilador escolhido, precisa ser aumentada em $20 \%$. Desta forma, a potência ideal para este tipo de ventilador é de $4,2 \mathrm{cv}$, que corresponde a $3100 \mathrm{~W}$.

A aplicação dos resultados obtidos ao longo deste trabalho resultará em um sistema de exaustão conforme a ilustração da Figura 5, onde é possível verificar a utilização dos componentes e das singularidades necessárias para realizar a montagem do sistema.

Com a utilização desse sistema de exaustão será possível captar as partículas resultantes do processo de soldagem, evitando a inalação dessas partículas por parte dos colaboradores. 


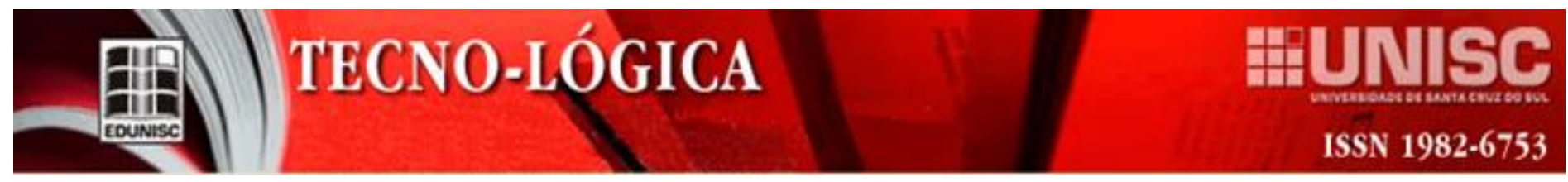

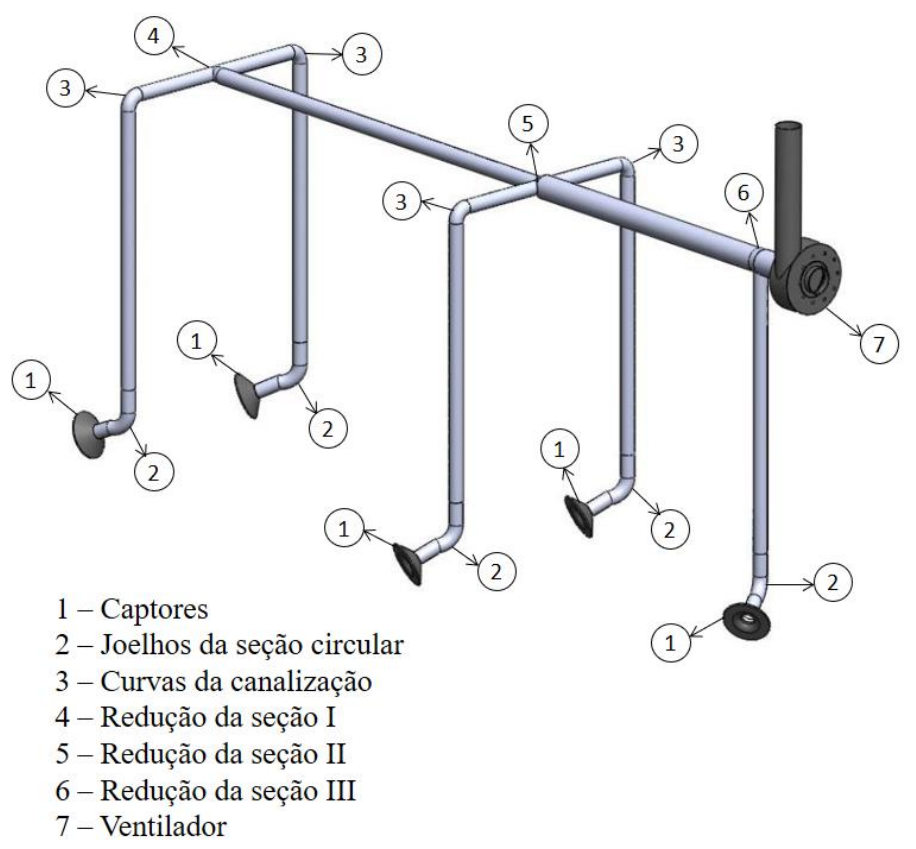

Figura 5 - Principais componentes do sistema de exaustão.

\section{Conclusões}

Diversas empresas possuem um sistema de ventilação natural que muitas vezes não é suficiente para realizar a coleta e o transporte adequado dos contaminantes resultantes desse processo, causando problemas de saúde entre os colaboradores envolvidos. Por isso, o processo de soldagem GMAW é amplamente utilizado por indústrias na união permanente dos componentes metálicos, e caracteriza-se por apresentar elevado nível de produtividade e permitir a utilização de dispositivos automatizados.

A sistemática adotada neste trabalho permitiu dimensionar os principais componentes, captores, tubulações, conexões e reduções de um sistema de exaustão, e também selecionar em catálogos técnicos o ventilador comercial mais adequado à empresa em estudo.

Neste estudo de caso, a definição dos parâmetros iniciais e as demais etapas do dimensionamento foram organizadas de forma sucessiva, a fim de facilitar a compreensão e o desenvolvimento do dimensionamento. Nesse método de cálculo, destaca-se a definição do tipo de partícula a ser retirada do ambiente, assim como a perda de carga resultante no sistema, o que acaba influenciando a escolha da potência necessária para que o ventilador realize a coleta e o transporte dos resíduos.

A identificação do ventilador ideal ocorreu em função do acréscimo de $20 \%$ em relação à potência calculada, compensando possíveis perdas. Assim, o ventilador atenderá a necessidade da empresa estudada.

\section{DIMENSIONING OF AN EXHAUST SYSTEM UTILIZED IN THE GMAW WELDING PROCESS IN AN AGRICULTURAL MACHINERY INDUSTRY}

\begin{abstract}
The joining of materials through the GMAW welding process is widely used in the agricultural machinery industry due to its characteristics that allow good level of productivity and process performance. However, the use of this welding process results in the generation of pollutants harmful to human health, also known as metallic fumes or toxic gases. Inhalation of these particles can cause health problems, such as decreased lung functions, and contribute to the emergence of various diseases. In this sense, it is necessary to use a system of capture and transport of these particles, avoiding the direct contact between the toxic gases and the operators involved in the process. In this context, this work aims to determine a methodology that allows carrying out the sizing of a system of capture and movement of these particles, from the choice of the captor to the definition of the type of fan to be used, as well as their respective characteristics. According to the calculation memorial discussed in this paper, it was possible to determine the ideal fan for this situation, taking into account the singularities used in the ducts, the type of contaminant and the loss of charge resulting from the collection and transportation of the toxic gases originated by the welding process.
\end{abstract}

Keywords: Metal fumes. Ventilation system. Forced ventilation. Welding process.

\section{Referências}

[1] BURGESS, W.A. Recognition of health hazards in industry: A review of materials and processes. Ed. By Burgess, WA, Wiley Interscience Publication, USA, 117-136, 1981.

[2] WAINER, E., S. D. BRANDI, F. D. H. MELLO. Soldagem - Processos e Metalurgia. Brasil, São Paulo: Edgard Blucher, 2004.

[3] OKUMURA, T., TANIGUCHI, C. Engenharia de soldagem e aplicações. Brasil, São Paulo: LTC, 1982.

[4] HEWITT, P. J. Strategies for risk assessment and control in welding: challenges for developing countries. The Annals of Occupational Hygiene 2001, pp: 295-298, 2001.

[5] ANTONINI, J. M. Health effects of welding. Crit. Rev. Toxicol., 33: 61-103, 2003.

[6] GARCIA, Regina Paula; SCOTT, Américo. Uma metodologia para análises comparativas da capacidade produtiva entre arames maciços (MIG/MAG) e tubulares (eletrodo tubular). Soldag. insp. (Impr.), São Paulo, v. 16, n. 2, p. 146155,2011 


\section{嘈. TECNO-LÓGICA}

[7] BAlthaZAR, A. P., M. ANDRADE, D. F. SOUZA, A. L. S. Braga. Occupational risks, working conditions and health of welders. Journal of nursing UFPE on line, 12 (4): 997-1008, 2018

[8] COSTA, Ê. C. Da. Ventilação. São Paulo: Edgard Blücher, 2005.

[9] OLIVEIRA, J. M De. Noções de ventilação Industrial. 2008. Disponível em: <http://ftp.demec.ufpr.br/disciplinas/TM120/VENTILACAO_INDUSTRIAL.pdf $>$. Acesso: 10 Jan. 2019.

[10] PASCOALI, Suzy. Soldagem Básica. Centro Federal de Educação Tecnológica de Santa Catarina de Araranguá. 2008

[11] GARCIA, Regina Paula; SCOTTI, Américo. Análise comparativa da geração de fumos entre arames maciços (GMAW) e tubulares (FCAW). Soldag. insp. (Impr.), São Paulo, v. 15, n. 2, p. 103-111, June 2010.

[12] FÜHR, T. A. Reconhecimento e avaliação dos riscos ambientais gerados nos processos de soldagem de uma empresa do segmento metal mecânico. Tese (Pós graduação/Engenharia de Segurança do trabalho) - Universidade Regional do Noroeste do Estado do Rio Grande do Sul, Ijuí. 2012.

[13] ANSCHAU, Léo Diel. Análise de fumos de soldagem, sistemas de proteção e desenvolvimento de protótipo para estudo da emissão de fumos de soldagem para processo Mig/Mag. 2010. 52f. Trabalho de conclusão de curso (Engenharia Mecânica) - Universidade Regional do Noroeste do Estado do Rio Grande do Sul - UNIJUÍ, Panambi, 2010.

[14] MARQUES, P. V.; MODENESI, P. J.; BRACARENSE, A. Q. Soldagem: fundamentos e tecnologia. 3. ed. Belo Horizonte: UFMG, 2009.

[15] MESQUITA, A. L. S.; GUIMARÃES, F. A.; NEFUSSI, N. Engenharia De Ventilação industrial. São Paulo: Edgard Blücher, 1977.

[16] CLEZAR, C. A; NOGUEIRA, A. C. R. Ventilação industrial. Florianópolis: UFSC, 1999.

[17] MACINTYRE, A. J. Ventilação Industrial E Controle Da Poluição. 2. ed. Rio de Janeiro: Guanabara, 1990.

[18] LISBOA, H. de M. Controle da poluição atmosférica: Ventilação Industrial. Montreal, 2007.

[19] OTAM. Ventiladores centrífugos RFS/RFD. Disponível em: <http://www.solerpalau.com.br/public/files/folders/d98c1pt_rfs_rfd_025_2011_j. pdf> Acesso em: 01 Dez. 2018.

[20] TUBOS, Oliveira. Catálogo de produtos. Disponível em: <http://www.tubosoliveira.com.br/wpcontent/uploads/2018/05/cat_a3_tubos_v03 _170704_ea.pdf>. Acesso em: 01 Dez. 2018. 